\title{
The role of dopamine in learning, memory, and performance of a water escape task
}

\author{
Victor H. Denenberg ${ }^{a}$, Douglas S. Kim ${ }^{\mathrm{a}, \mathrm{b}}$, Richard D. Palmiter ${ }^{\mathrm{a}, \mathrm{b}, \mathrm{c}, *}$ \\ ${ }^{a}$ Department of Biochemistry, University of Washington, Box 357370, Seattle, WA 98195-7370, USA \\ ${ }^{\mathrm{b}}$ Molecular and Cellular Biology Program, University of Washington, Box 357370, Seattle, WA 98195-7370, USA \\ ${ }^{\mathrm{c}}$ Howard Hughes Medical Institute, University of Washington, Box 357370, Seattle, WA 98195-7370, USA
}

Received 1 February 2003; received in revised form 1 May 2003; accepted 8 May 2003

\begin{abstract}
Dopamine-deficient (DD) mice have selective inactivation of the tyrosine hydroxylase gene in dopaminergic neurons, and they die of starvation and dehydration at 3-4 weeks of age. Daily injections of L-DOPA (50 mg/kg, i.p.) starting $\sim 2$ weeks after birth allow these animals to eat and drink enough for survival and growth. They are hyperactive for $6-9 \mathrm{~h}$ after receiving L-DOPA and become hypoactive thereafter. Because these animals can be tested in the presence or absence of DA, they were used to determine whether DA is necessary for learning to occur. DD mice were tested for learning to swim to an escape platform in a straight alley in the presence (30 min after an L-DOPA injection) or absence (22-24 h after an L-DOPA injection) of dopamine. The groups were split $24 \mathrm{~h}$ later and retested $30 \mathrm{~min}$ or 22-24 $\mathrm{h}$ after their last L-DOPA injection. In the initial test, DD mice without dopamine showed no evidence of learning, whereas those with dopamine had a learning curve similar in slope to controls but significantly slower. A retest after $24 \mathrm{~h}$ showed that DD mice can learn and remember in the absence of dopamine, leading to the inference that the lack of dopamine results in a performance/motivational decrement that masks their learning competence for this relatively simple task.
\end{abstract}

(c) 2003 Elsevier B.V. All rights reserved.

Keywords: Aversive learning; Tyrosine hydroxylase; Performance deficits; Dopamine-deficient mice

\section{Introduction}

It is well established that dopamine (DA) plays a central role in aspects of incentive motivation [1,7-11]. However, the nature of that role is less certain. From electrophysiological studies of monkeys in learning situations, Schultz [11] concludes that midbrain DA neurons are likely to show phasic activation following rewards and reward-predicting stimuli. However, studies with rats using DA agonists and antagonists, and 6-hydroxydopamine (6-OHDA) neurotoxic lesions lead to different interpretations. After reviewing the literature, Salamone [7] states that "involvement of DA systems in instrumental behavior is not selective for those procedures involving positive reinforcement" (p. 125). Berridge and Robinson [1] from their own experimental findings and literature review, conclude that mesolimbic and neostriatal DA systems do not mediate the hedonic impact of rewarding

\footnotetext{
* Corresponding author.

E-mail addresses: denenberg@mac.com (V.H. Denenberg), palmiter@u.washington.edu (R.D. Palmiter).
}

stimuli, nor do they mediate reward learning and associative prediction. Instead, they state that DA systems are necessary for a component of motivation and reward called incentive salience that involves the "wanting" of incentives but is independent of whether the animal does or does not "like" the incentive.

An equally sharp theoretical distinction is seen when the area of aversive learning is examined. In monkeys, only $14 \%$ of DA neurons respond to primary aversive stimuli, whereas $75 \%$ respond to food; for a conditioned stimulus (CS), $11 \%$ of DA neurons respond to an aversive CS compared to $55-70 \%$ for a positive CS [11]. This asymmetry led Schultz [11] to conclude that "phasic activations of DA neurons report preferentially environmental events with rewarding value..." (p. 242). However, in rats, this bias is not seen, and DA appears to be equally involved in appetitive and aversive learning [1,7]. Thus, an increase in DA levels is brought about by food reward and drugs of abuse; and also by a variety of aversive stimuli including electric shock, restraint, cold, tail-pinch, forced exercise, anxiogenic beta-carboline drugs, and CS associated with shock stress 
(see Ref. [7] for review and references). Further, taste aversion and active avoidance learning are impaired by a variety of DA antagonists; this also appears to be the case for passive learning, though the evidence is not as definitive [1,7].

The contradictory conclusions reached by these researchers probably reflect a complex combination of species and procedural differences. A salient difference is that the studies in monkeys reflect transient changes in activity of DA neurons in response to food or cues, which produce equally transient changes in striatal DA levels, whereas the changes in DA measured in rats in response to aversive stimuli reflect relatively long-lasting changes in dopaminergic tone in the striatum. In addition, there is a deeper distinction. The electrophysiological studies with monkeys ask the question: Is DA correlated with the occurrence of reward and aversive learning? In contrast, the 6-OHDA lesion studies with rats ask: Is DA necessary to mediate reward and aversive learning? Viewed from this perspective, both the monkey and the rat studies may be equally valid. That is, under normal physiological conditions activation of DA neurons may be highly associated with the occurrence of reward learning but only be modestly correlated with aversive learning; yet both appetitive and aversive learning can still occur in the absence of DA.

The two questions noted above demand different procedures. To study association, an intact organism is needed. However, to determine necessity, one has to eliminate a vital part of the organism either irreversibly (e.g. destroying part of the brain via 6-OHDA) or temporarily (e.g. use of DA agonists and antagonists). Note also that the question "Can a DA-deficient mouse learn?" does not involve comparisons between control and experimental animals. As an example, Taghzouti et al. [13] studied passive avoidance learning in rats injected with 6-OHDA in the nucleus accumbens and sham controls. The controls had a greater latency than the lesioned rats to enter a dark compartment after previously receiving electric shock in that compartment, but the lesioned animals also learned the association by increasing their latency from 6 to $75 \mathrm{~s}$. In that same paper Taghzouti et al. reported that an independent group of lesioned rats did not differ from shams in learning to run down a straight alley to obtain food, though the lesioned animals did take longer to extinguish the response. Thus, in both studies lack of DA in the accumbens did not prevent learning from occurring, although the 6-OHDA lesions clearly impaired the performance of the rats.

In addition to lesion and pharmacological procedures, it is now possible to eliminate DA by genetic manipulations. Dopamine-deficient (DD) mice [16] cannot synthesize tyrosine hydroxylase $(\mathrm{TH})$ in dopaminergic neurons but can do so in noradrenergic neurons. Zhou and Palmiter [16] reported that the pups were normal at birth but their growth curve began to taper off around postnatal days 10-15 (P10-P15) and all were dead by P28. A daily injection of L-DOPA $(50 \mathrm{mg} / \mathrm{kg}$, i.p.) is sufficient to rescue the animals $[4,12,16]$.
In comparison to wildtype (WT) controls, DD mice become hyperactive within $15 \mathrm{~min}$ after receiving L-DOPA, continue their activity for 6-9h, and consume approximately $90 \%$ of their daily food and water intake during this interval [12]. Over the 24-h day, WT and L-DOPA treated DD mice ingest comparable amounts of food and water, but the mutants are seven times more active [12].

Szczypka et al. [12] analyzed catecholamine content in the brains of DD mice before and after L-DOPA treatment. Striatal analyses of DA content revealed that, compared to WT mice, DD mice have $\sim 1 \%$ of control levels of DA $24 \mathrm{~h}$ after their last L-DOPA injection [12]. L-DOPA brings the DA content up to $9.1 \%$ of WTs, which is sufficient to generate the hyperactivity and appropriate food and water consumption. Nine hours after an L-DOPA injection striatal DA drops to $3.8 \%$ of normal, and this is when feeding and activity decline [12]. Thus, over a 24-h L-DOPA injection cycle, striatal DA ranges from 9\% of WT to 1\%. In parallel, the animal's behavior ranges from hyperactivity and adequate food consumption following L-DOPA to hypoactivity and aphagia $20-24 \mathrm{~h}$ later $[3,12,16]$. Therefore, by giving DD mutants a cognitive task immediately before or after an L-DOPA injection and retesting them $24 \mathrm{~h}$ later, again before or after an L-DOPA injection, we can study learning and memory in the presence or absence of DA.

\section{Methods and materials}

\subsection{Subjects}

Mice lacking TH $\left(\mathrm{Th}^{-/-}\right)$die as embryos due to lack of norepinephrine (NE) $[15,16]$. To remedy the developmental requirement for $\mathrm{NE}$, the $T h$ gene was targeted to the $D b h$ allele, which restores NE synthesis in all cells that normally make DA as a precursor of NE or epinephrine but not in dopaminergic neurons. DD mice (previously called $\mathrm{DA}^{-/-}$ mice) were generated by breeding $T h^{-/-}, D b h^{T h /+}$ compound heterozygotes on a mixed C57BL/ $6 \times 129 / \mathrm{Sv}$ genetic background with each other. DD mice (about one-seventh of total offspring) were identified at about 2 weeks of age by their small size and responsiveness to L-DOPA. Control mice for these experiments had at least one intact $T h$ allele and one intact $D b h$ allele which is sufficient to produce nearly normal levels of DA and NE, respectively $[6,14]$.

The mice were maintained on a 12-h light cycle; lights on at $07: 00 \mathrm{~h}$. They received daily injections of $50 \mathrm{mg} / \mathrm{kg}$, i.p. L-DOPA [12] starting $\sim 2$ weeks after birth. L-DOPA injections were at $\sim 10: 00 \mathrm{~h}$ and testing began $\sim 30 \mathrm{~min}$ later. Mice that were tested without L-DOPA were given their daily injection after termination of testing, between 12:00 and 14:00 $\mathrm{h}$. The mice used for these studies were 59-306 days old and were either littermates or closely matched by age. The mice were maintained and used according to guidelines of the National Institutes of Health and the University of Washington Animal Care Committee. 


\subsection{Apparatus and procedure}

A galvanized tin straight alley, $61.0 \mathrm{~mm}$ long and $12.7 \mathrm{~mm}$ wide, was placed inside a tub of water at room temperature $\left(23^{\circ} \mathrm{C}\right)$. A visible black circular platform $(7.6 \mathrm{~mm}$ diameter $)$ elevated $1.3 \mathrm{~mm}$ above the water level was placed at one end of the alley. The platform was covered with fine wire mesh that the mouse could easily grasp to climb atop the platform.

A mouse was released into the water at the end opposite the platform, and a timer was started at the same time. When the mouse contacted the platform with both front feet, the timer was stopped. It remained on the platform for $20 \mathrm{~s}$ and was then returned to its heated cage. A maximum of $60 \mathrm{~s}$ was allowed. In those few instances where an animal was still swimming after $60 \mathrm{~s}$, it was guided to the platform by the experimenter and left there for $20 \mathrm{~s}$.

After all squad members had their first trial, the second trial was begun. The mice were tested for five trials. All mice were then returned to the colony room. A day later, at approximately the same time, the mice were retested for five trials, following the procedure described above.

\subsection{Experimental rationale}

The major problem in working with the DD mouse is its hypoactivity near the end of the 24-h L-DOPA cycle. This clearly impairs performance in many areas, including learning tasks. However, lack of performance does not necessarily mean lack of learning [2]. Therefore it is necessary to design experiments to separate performance factors from cognitive processes, as shown in Tables 1 and 2.

Table 1 employs two DD groups to study original learning (called Test). DD mice were given a learning task approximately a half-hour after an L-DOPA injection (DD/Y) or 22-24 h after their last L-DOPA injection (DD/N). Thus, the first group is exposed to a learning situation in the presence of DA, while the second group is tested in the absence of DA. If the DD/N group generates a learning curve (defined as a decrease in time over trials), then we conclude that learning is possible in the absence of DA, and our question is answered. However, if this group does not show evidence

Table 1

Experimental design to determine if DA is needed for learning: Test data

\begin{tabular}{lll}
\hline $\begin{array}{l}\text { L-DOPA } \\
\text { present at Test }\end{array}$ & Group & Treatment \\
\hline Yes & DD/Y & L-DOPA injection \\
& & 30 min prior to Test \\
No & DD/N & Last L-DOPA injection \\
& & 22-24h prior to Test \\
\hline
\end{tabular}

Specific comparisons. Learning: Do DD/N mice have a learning curve? If so, conclude DA is not needed for learning. If not, no conclusion is possible because failure to perform may or may not reflect failure to learn. L-DOPA rescue: Do DD/Y mice have a learning curve? If so, conclude L-DOPA is effective in rescuing DD mice.
Table 2

Experimental design to determine if DA is needed for learning and memory: Test and Retest data

\begin{tabular}{lll}
\hline L-DOPA present at Test & \multicolumn{2}{l}{ L-DOPA present at Retest } \\
\cline { 2 - 3 } & No & Yes \\
\hline Yes-DD/Y & DD/YN & DD/YY \\
No-DD/N & DD/NN & DD/NY
\end{tabular}

Specific comparisons. Learning: DD/NY Retest data vs. DD/Y Test data. If nothing was learned during the Test phase in absence of DA, DD/NY Retest curve should not differ from the DD/Y Test curve. However, if $\mathrm{DD} / \mathrm{NY}$ is superior to $\mathrm{DD} / \mathrm{Y}$, conclude learning occurred in the absence of DA. Memory: DD/YN vs. DD/NN Retest data. If $\mathrm{DD} / \mathrm{YN}$ is superior to $\mathrm{DD} / \mathrm{NN}$, conclude experience during Test phase in presence of DA is remembered during Retest phase without DA. Therefore, DA not needed to remember a previously learned event. L-DOPA rescue: Does DD/YY show a good Retest curve? If so, conclude L-DOPA is effective for memory retention.

of learning, while group $\mathrm{DD} / \mathrm{Y}$ does, no conclusion can be drawn since the failure to improve over trials may represent an inability to perform, rather than an inability to form the neural associations required for learning.

The 24-h Retest data (Table 2) provides two more opportunities to separate performance factors from cognitive processes. The mice from each of the DD groups in Table 1 are split, half being retested in the absence of DA (DD/YN and $\mathrm{DD} / \mathrm{NN}$ ) and half in the presence (DD/YY and DD/NY). Consider, first, the DD/N mice; if they failed to show a learning curve during the Test phase, this could be because (1) of a cognitive failure (i.e. they learned nothing during the Test experience in the absence of DA), or (2) learning occurred but could not be expressed because of performance deficits. If the first argument is correct, then their learning curve on Retest in the presence of DA (Group DD/NY) should not differ significantly from the learning curve of the $\mathrm{DD} / \mathrm{Y}$ mice. However, if the second alternative is correct, then their learning curve during Retest is expected to be superior to the original learning curve of the $\mathrm{DD} / \mathrm{Y}$ mice.

The above comparison addresses the question of whether DA is needed for learning to occur. The next comparison asks whether DA is needed to remember an already-learned response. The groups $\mathrm{DD} / \mathrm{YN}$ and $\mathrm{DD} / \mathrm{NN}$ are both retested in the absence of DA. They differ in that the former group learned during the Test phase shortly after an L-DOPA injection. If DA must be present to remember a learned response, then the two groups should not differ statistically. If, however, $\mathrm{DD} / \mathrm{YN}$ mice are superior to $\mathrm{DD} / \mathrm{NN}$ mice, we will conclude that DA is not needed to remember a previously learned response.

The difficulty in implementing the study outlined in Tables 1 and 2 is that DD mice are essentially inactive at the nadir of their L-DOPA. However, in water they did as well as WT mice in the forced-swim test [12]. We have observed that DD mice placed into water will actively swim, they almost never float, and they are capable of making instrumental responses. Therefore, they were tested in 
a straight alley swimming task. Their time to swim to a visible platform was measured.

In addition to the DD mice described above, WT controls were also tested. The major reason was to compare their Test and Retest curves to those of DD mice receiving L-DOPA prior to testing to see how effectively L-DOPA rescued DD mice. A subset of WT mice were given L-DOPA injections prior to Test and Retest and compared to non-injected controls. No significant differences were found and the mice were pooled into a single WT control group.

\section{Results}

\subsection{Statistical analyses}

The data were evaluated by use of analysis of variance (ANOVA).

Preliminary analyses determined that sex was not significant as a main effect or in interaction and was not included in the final analyses. Age was significantly correlated with swimming time, with older animals taking longer to reach the platform (intraclass correlation $=0.33, P<0.01$ ). Therefore Age was used as a covariate in the ANOVAs.

Fig. 1 shows the Test (learning) curves for the three groups, and the Retest (memory) curves for the five groups.

\subsection{Test phase}

\subsubsection{Learning}

The DD/N mice did not show a learning curve during the Test phase. Indeed, their times increased over trials and approached significance $(P<0.10)$.
DD/Y mice showed a significant learning curve with swim times decreasing over trials $[F(4,56)=3.17, P<0.03]$.

\subsection{2. $\mathrm{L}-D O P A$ rescue}

A comparison between the WT and the DD/Y groups found a significant main effect $[F(1,44)=8.29, P<0.01]$ but no interaction. Thus, L-DOPA rescued the DD mice in that they showed a learning curve with the same slope as the WT mice, even though they took longer on average to reach the visible platform.

\subsection{Retest phase}

\subsubsection{Learning}

The Test curve of the DD/Y mice was compared to the Retest curve of the DD/NY group. See Fig. 2. Both groups were tested in the presence of DA, but the DD/NY mice had had prior learning exposure in the absence of DA. If the two curves are comparable, then the prior learning exposure was without effect. However, the ANOVA found a significant Group effect: the DD/NY took less time to reach the platform than the DD/Y mice $[F(1,24)=4.39, P<0.023$, one-tailed test]. There was a significant Trials effect $(P<0.001)$, but the Groups $\times$ Trials interaction was not significant.

\subsubsection{Memory}

Groups DD/YN and DD/NN were both evaluated during Retest in the absence of L-DOPA. If DA is needed to remember a previously learned behavior, then the two groups should not differ. However, there is a striking difference: the former group showed improvement with trials while the DD/NN group increased its time to get to the platform over trials [Group $\times$ Trials Linear interaction $F(1,12)=7.23$, $P<0.01$, one-tailed test].

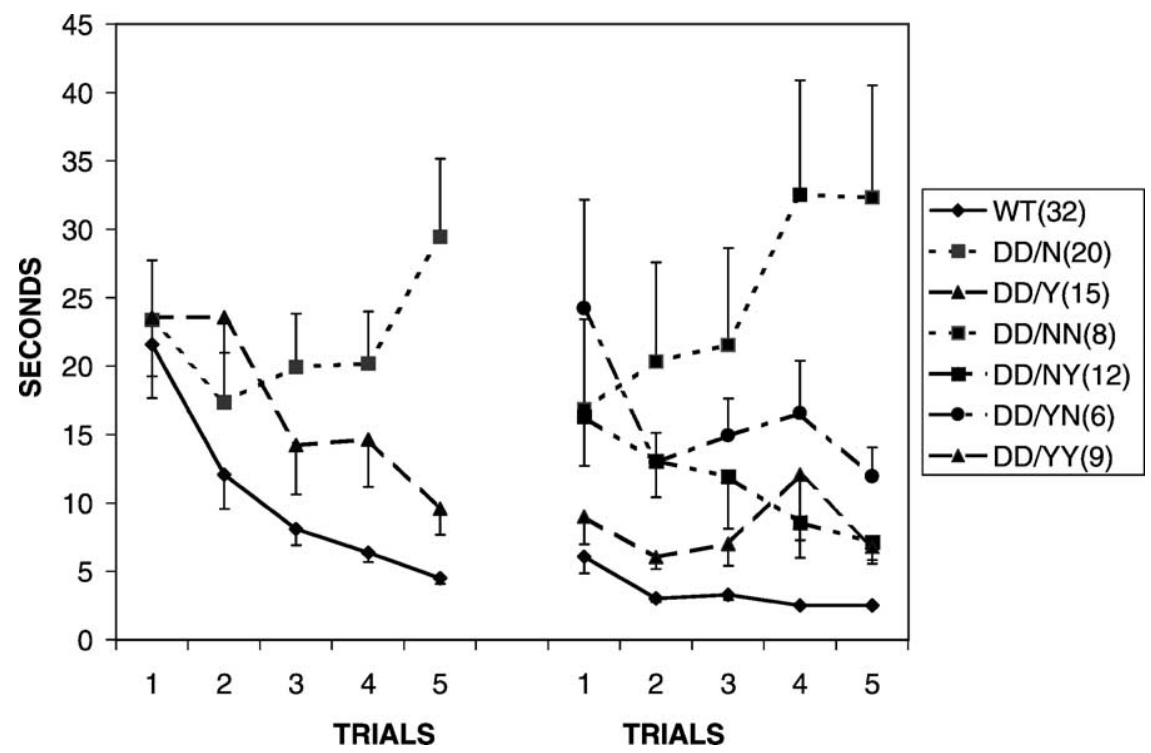

Fig. 1. Means and S.E.s (in seconds) to swim to platform over five trials during Test phase (3 groups on left) and Retest phase (5 groups on right). See text for definitions of groups. $N$ is given in parentheses. 


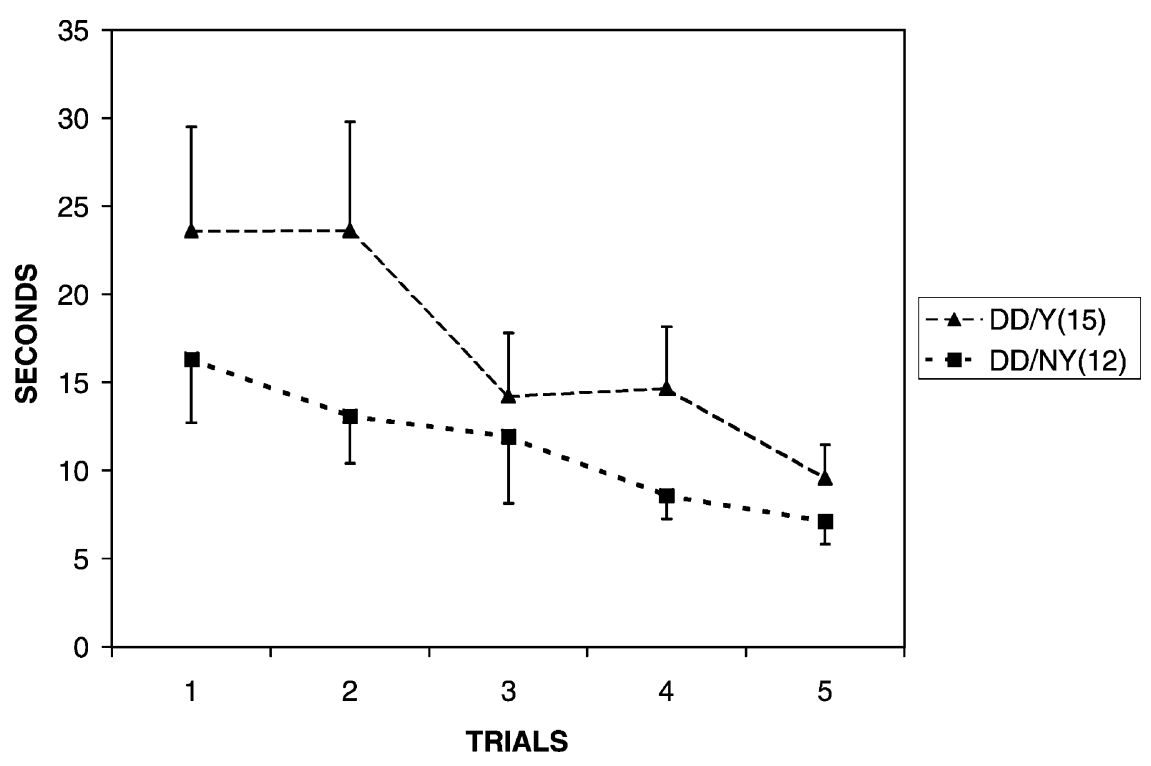

Fig. 2. Comparison of Test phase curve of DD/Y group with Retest phase curve of DD/NY group. $N$ is given in parentheses.

\subsubsection{DA rescue}

The Retest data of the WT and DD/YY groups were compared. The WT mice had shorter escape times $[F(1,38)=$ 15.76, $P<0.001]$, and the Groups $\times$ Trials interaction was significant $[F(4,156)=2.58, P<0.04]$.

\section{Discussion}

To evaluate learning in the absence of DA, the Test curve of the $\mathrm{DD} / \mathrm{Y}$ group was compared to the Retest curve of the DD/NY group. These curves were obtained under comparable physiological conditions. The groups differed only in that the DD/NY group had failed to show any evidence of learning in the Test phase. The superior scores of the $\mathrm{DD} / \mathrm{NY}$ mice during the Retest phase indicate that the lack of DA during the Test phase, though it prevented animals from performing efficiently, did not prevent learning from occurring. It might be argued that L-DOPA administered after the Test phase facilitated memory consolidation in the $\mathrm{DD} / \mathrm{NY}$ group. However, the reverse trend of the DD/NN mice counters this argument.

Our memory test involved the two groups retested in the absence of DA (DD/NN and DD/YN). These groups would only differ if the prior learning experience of the DD/YN mice was carried over to the Retest phase, which was observed.

The Retest data were critical in allowing us to dissociate performance deficits from the positive findings concerning learning and memory. These data reflect a mixture of 24-h long-term memory requiring protein synthesis plus short-term memory from trial to trial. This suggests that long-term memory and protein synthesis can function in the absence of DA, at least for a relatively simple aversive learning task.
In summary, DA is not needed for the acquisition or storage of some types of aversive learning but is needed for the effective performance of such a response. This conclusion concerns plastic neural circuits and parallels a similar conclusion of Zhou and Palmiter [16] who found that DA is needed for the motivated behaviors of moving, drinking, and eating, but is not required to establish the neural circuits that control these behaviors. However, the long-term presence of dopaminergic input is required to dampen the responsiveness of postsynaptic DA receptors to the neurotransmitter itself [3]. These findings are also consistent with the conclusions of Salamone [7] and Berridge and Robinson [1] that aversive learning can occur in the absence of DA.

Nishii et al. [5] studied avoidance learning using a DD mouse that is similar, in principle, to the ones we have been analyzing. They tested their mutants between 21 and 27 days of age for one-way avoidance learning to a tone stimulus in a shuttlebox where the mice received shock if they did not move out of a dark chamber into a lighted one. Their mice were never injected with L-DOPA, but had brain DA levels that were $34 \%$ of WT levels. Although moribund, some of the mice survived through the testing sessions, but Nishii et al. [5] reported that they all died by 30 days. Both the mutants and WT mice showed significant improvement in the number of avoidance responses over time with the WT mice having significantly better scores. They claimed that there was a difference in the shape of the two learning curves favoring the WT mice, but they presented no statistical evidence to support that assertion. Their study involved a relatively complex foot-shock paradigm on land, whereas we used a relatively simple swimming response to a visible platform, yet both studies found that mice without DA were able to learn an aversive response regardless of task complexity. It will be interesting in the future to examine our DD mice in a variety of other learning tasks, including some 
that are more aversive than the water escape task described here.

\section{Acknowledgements}

We thank Kristen Nagata and Nora Meneses for animal care and Dr. Robert McGivern for his comments.

\section{References}

[1] Berridge KC, Robinson TE. What is the role of dopamine in reward: hedonic impact, reward learning, or incentive salience? Brain Res Brain Res Rev 1998;28:309-69.

[2] Hilgard ER. Theories of learning. New York: Appleton-CenturyCrofts; 1948.

[3] Kim DS, Froelick GJ, Palmiter RD. Dopamine-dependent desensitization of dopaminergic signaling in the developing mouse striatum. J Neurosci 2002;22:9841-9.

[4] Kim DS, Szczypka MS, Palmiter RD. Dopamine-deficient mice are hypersensitive to dopamine receptor agonists. J Neurosci 2000;20:4405-13.

[5] Nishii K, Matsushita N, Sawada H, Sano H, Noda Y, Mamiya T, et al. Motor and learning dysfunction during postnatal development in mice defective in dopamine neuronal transmission. J Neurosci Res 1998;54:450-64.

[6] Rios M, Habecker B, Sasaoka T, Eisenhofer G, Tian H, Landis S, et al. Catecholamine synthesis is mediated by tyrosinase in the absence of tyrosine hydroxylase. J Neurosci 1999;19:3519-26.

[7] Salamone JD. The involvement of nucleus accumbens dopamine in appetitive and aversive motivation. Behav Brain Res 1994;61:117-33.

[8] Schultz W. Dopamine neurons and their role in reward mechanisms. Curr Opin Neurobiol 1997;7:191-7.

[9] Schultz W. Multiple reward signals in the brain. Nat Rev Neurosci 2000;1:199-207.

[10] Schultz W. Reward signaling by dopamine neurons. Neuroscientist 2001;7:293-302.

[11] Schultz W. Getting formal with dopamine and reward. Neuron 2002;36:241-63.

[12] Szczypka MS, Rainey MA, Kim DS, Alaynick WA, Marck BT, Matsumoto AM, et al. Feeding behavior in dopamine-deficient mice. Proc Natl Acad Sci USA 1999;96:12138-43.

[13] Taghzouti K, Simon H, Louilot A, Herman JP, Le Moal M. Behavioral study after local injection of 6-hydroxydopamine into the nucleus accumbens in the rat. Brain Res 1985;344:9-20.

[14] Thomas SA, Marck BT, Palmiter RD, Matsumoto AM. Restoration of norepinephrine and reversal of phenotypes in mice lacking dopamine beta-hydroxylase. J Neurochem 1998;70:2468-76.

[15] Thomas SA, Matsumoto AM, Palmiter RD. Noradrenaline is essential for mouse fetal development. Nature 1995;374:643-6.

[16] Zhou QY, Palmiter RD. Dopamine-deficient mice are severely hypoactive, adipsic, and aphagic. Cell 1995;93:1197-209. 